\title{
Impact of psychosocial intervention on prognosis of cardiac surgery patients
}

\author{
Marlyn Thomas Savio, ${ }^{1}$, Meena Hariharan ${ }^{2}$ \\ ${ }^{1}$ Freelance Psychologist; ${ }^{2}$ Professor, Centre for Health Psychology, University of Hyderabad, India
}

\begin{abstract}
The study examined the impact of two types of psychosocial intervention on prognosis and psychological distress of patients subjected to Coronary Artery Bypass Grafting (CABG), and investigated whether such impact was direct or mediated. A sample of 300 participants (243 men and 57 women; mean age: 56.1 years) was recruited into three groups. One group received a unique psycho-educational intervention called Programme for Affective and Cognitive Education (PACE), the second group was given a Relaxation intervention (Guided Imagery), and the third group had only standard medical treatment (Control). The PACE and Relaxation interventions were administered to patients alongside standard treatment a day before $\mathrm{CABG}$ as well as a day before discharge from hospital. Prognosis was assessed six weeks after discharge. Psychological distress was measured at baseline, week after discharge, and six weeks after discharge. Analyses of variance revealed a significant effect of groups on prognosis and a significant group $\times$ time interaction for psychological distress. The highest prognosis after $\mathrm{CABG}$ and the lowest psychological dis-
\end{abstract}

Correspondence: Marlyn Thomas Savio, Freelance Psychologist, India. E-mail: marlyn.thomas@gmail.com

Key words: Coronary artery bypass grafting; coronary artery disease; intervention; prognosis; psychological distress.

Acknowledgements: We are grateful to all the participants, doctors, hospital authorities and staff, and caregivers for their respective contributions. Thanks to the Indian Council of Medical Research (ICMR) for funding the PhD position of Marlyn Thomas Savio.

Contributions: MTS: Literature review, data collection, analyses and interpretations, report writing; MH: Study conceptualisation and design, review, analyses, interpretations.

Conflict of interest: The authors declare no conflict of interest.

Received for publication: 7 February 2020

Revision received: 24 July 2020.

Accepted for publication: 5 October 2020.

This work is licensed under a Creative Commons AttributionNonCommercial 4.0 International License (CC BY-NC 4.0).

(C) Copyright: the Author(s), 2020

Licensee PAGEPress, Italy

Health Psychology Research 2020; 8:8887

doi:10.4081/hpr.2020.8887 tress were found in the PACE group. Path analyses indicated that the PACE and Relaxation interventions negatively impacted psychological distress which in turn predicted higher prognosis. Only the PACE intervention additionally had a positive direct impact on prognosis. Both psychosocial interventions helped enhance the prognosis of patients after CABG primarily by reducing psychological distress. PACE emerged as a more effective intervention than Relaxation.

\section{Introduction}

Coronary Artery Bypass Grafting (CABG) is a common major surgery performed on patients suffering from the world's leading cause of death and disability - Coronary Artery Disease or CAD (Gaziano, Bitton, Anand, Abrahams-Gessel, \& Murphy, 2010; World Health Organization, 2015). Patients awaiting such invasive medical procedures typically experience psychological distress, which refers to the presence of sub-clinical anxiety and depressive symptoms (Drapeau, Marchand, \& Beaulieu-Prévost, 2012). Their co-existence with an already burdensome medical condition (e.g., CAD) compels attention and intervention for the patient (Thomas, Hariharan, \& Rana, 2016). There is adequate empirical evidence on the significant association between preCABG and post-CABG distress (Chaudhury, Saini, Bakhla, \& Singh, 2016; Douki et al., 2011), the worsening of CAD pathology due to distress-induced hyperactivation of the sympathetic pathway (Wulsin, 2012), and the interference of distress-related cognitive biases and lethargy in recovery-related health behaviours (Redelmeier, Rozin, \& Kahneman, 1993; Upton \& Solowiej, 2010). The credibility of a treatment rests upon the prognosis of patients, which refers to the prospects of the patient in the long run (Hilden \& Habbema, 1987). CABG endows good clinicallydefined prognosis through relieving symptoms (e.g., angina and shortness of breath) and increasing survival (Miller, 1977; Sipahi, Akay, Dagdelen, Blitz, \& Alhan, 2014). However, patients' selfreports of health have not been equally satisfying, and have in fact pointed at their distress on account of feeling uncertain, isolated and functionally restricted after CABG (Parker \& Adams, 2008). For one, patients awaiting surgery and discharge lack information or are misinformed about the procedure and its aftermath (Fathi et al., 2014; Martinsen \& Moen, 2010). Distress and poor prognosis may be an outcome of this knowledge lacuna (Broadbent, Petrie, Ellis, Ying, \& Gamble, 2004). This points to the relationship between the cognitive and affective states. Furthermore, research evidence has established the association between psychological distress and outcomes of patients with CAD - self-rated health and psychosocial symptoms were better predictive of future casualties and resumption of normal routine than medical prognosis (Mallik et al., 2005; Pedersen et al., 2011). Psychosocial intervention that addresses patients' psychological and social concerns 
through applying psychological principles and techniques has to be integrated into the care-plan for patients undergoing CABG (Protogerou et al., 2015; Smith, 2012). Since psychological distress reduction is the principal target, relaxation therapy is apt. It non-invasively generates positive emotions that counter the negative state of mind in the distressed patient (van Dixhoorn \& White, 2005). Alternatively, psycho-educational intervention can disseminate credible information as well as, modify beliefs and behaviours and enable patients for active health management (Colom, 2011). The current study designed a unique intervention called Programme for Affective and Cognitive Education (PACE) to extend knowledge and emotional support that may assuage distress and also promote health in patients subjected to CABG. The chief aim was to examine the differential impact of PACE and Relaxation, in comparison to biomedical treatment (standard hospital care). The design adopted was quasi-experimental, specifically a pretest-posttest non-equivalent control groups design. Three hypotheses were formulated: i) Exposure to the PACE or Relaxation intervention would enhance prognosis in patients undergoing $\mathrm{CABG}$, ii) There would be differential effects of the psychosocial interventions on psychological distress across time, iii) Psychosocial intervention would follow a pathway through psychological distress in impacting prognosis.

\section{Materials and Methods}

\section{Participants}

The study sought patients in the 25-70 years age-range, who were willing to participate and had a provision to use Compact Disc (CD) and Digital Video Disc (DVD) at home. Those with history of mental illness or other co-morbidities besides hypertension and diabetes were excluded. A purposive sample of 322 patients undergoing $\mathrm{CABG}$ for the first time were recruited from four hospitals in Hyderabad, India. Twenty-two among these patients were lost at different time-points of the study (either had not completed intervention or follow-up), leaving 300 (243 men and 57 women) in the final sample with a mean age of 56.05 years $(S D=7.72)$. Patients were sequentially assigned to one of the three groups in the order of PACE, Relaxation, and Control. Each group had 100 participants (81 men and 19 women). Group-wise characteristics are presented in Table 1. Results of one-way Welch ANOVA indicated that there were no significant differences in terms of age, years of education and psychological distress, suggesting homogeneity among the groups.

\section{Tools}

\section{Hospital Anxiety and Depression Scale (HADS)}

The 14-item HADS (Zigmond \& Snaith, 1983) measured the level of anxiety and depression typically experienced by medical patients during the preceding week. Items were presented on a 4- point scale in the range of $0-3$. When taken together, the 14 items represented the patient's overall psychological distress. Higher score indicated higher level of psychological distress. A review of over 700 papers that reported using HADS indicated that the mean Cronbach's alpha was 0.83 and 0.82 for the anxiety and depression sub-scales respectively (Bjelland, Dahl, Haug, \& Neckelmann, 2002). During pilot testing, the mean Cronbach's alpha values were found to be 0.75 (anxiety), 0.65 (depression), and 0.80 (total scale).

\section{Biopsychosocial Prognosis Scale for CABG (BIPROSCAB)}

Comprising 25 items, BIPROSCAB (Hariharan, Thomas, \& Rana, 2017) measured the level of prognosis during the month after CABG. The items relating to bio-physiological symptoms and psychosocial issues after surgery were presented on a 5-point scale of frequency of experience. BIPROSCAB had nine dimensions - 'post-CABG affect state', 'post-CABG anxiety', 'postCABG physical pain', 'discomfort in surgical sites', 'worry about return to normalcy', 'discomfort in the leg', 'CABG bio-social byproducts', 'constraints in socialising', and 'infection and interference to routine life'. Higher scores implied higher levels of prognosis overall and/or dimension-wise. The reliability coefficient of the scale was .73 .

Participants' demographic details were also obtained.

\section{Interventions}

\section{Programme for Affective and Cognitive Education (PACE)}

PACE was a specifically developed psycho-educational intervention to address the cognitive (knowledge) and affective (emotional) concerns of patients electing to have CABG. It was designed in video format, containing discussions among a cardiothoracic surgeon (who elucidated on $\mathrm{CAD}$, need for CABG, benefits and risks of surgery, and post-operative care), a peer patient with a history of CABG (who recounted the practical, emotional and physical experiences one witnesses during the period), and a health psychologist (who drew attention towards relevant psychosocial aspects, viz., availing social support, managing distress, and coping effectively). Two separate recordings were made in order to match the gender of the peer patient with that of the participant. PACE was divided into two 20-minute modules-presurgery and pre-discharge.

\section{Relaxation}

The technique of Guided Imagery was used herein. The 18minute audio module, facilitated by a clinical psychologist, provided suggestions to relax the patient somatically and affectively by helping her or him visualise the scenery of a hill station. Suggestions were narrated to prompt images and experiences in the mind of the participant from her or his exclusive mental repertoire. Such visualisation of suggestions was meant to further relax the breathing pattern, heart rate and blood pressure, thereby lowering distress.

Table 1. Characteristics of the study groups at baseline.

\begin{tabular}{lcccc} 
& & $M($ SD $)$ & & \\
& PACE & Relaxation & Control & $F(2,197.3)=0.52(p>0.05)$ \\
Age (years) & $56.44(7.12)$ & $55.40(8.16)$ & $56.31(7.86)$ & $F(2,197.8)=1.09(p>0.05)$ \\
Education (years) & $8.97(5.29)$ & $10.02(5.18)$ & $9.25(4.90)$ & $F(2,197.2)=1.13(p>0.05)$ \\
\hline Psychological distress (HADS) & $8.80(4.62)$ & $9.03(4.73)$ & $9.84(5.39)$ & \\
\hline
\end{tabular}




\section{Procedure}

Approval of the institutional ethics committee was obtained. Patients who provided written informed consent were recruited a day before CABG in their ward/room. HADS was individually administered. Patients were sequentially assigned to one of the groups in the order of PACE, Relaxation, and Control. Those in the PACE and Relaxation groups individually received their respective intervention. Patients underwent $\mathrm{CABG}$ on the following day. A day prior to discharge from hospital, patients in the PACE and Relaxation groups were given their respective interventional module. A CD of Guided Imagery was handed over to each patient in the Relaxation group while those in the PACE group received a DVD of the pre-discharge PACE module. Reminders were given up to six weeks after discharge to the Relaxation group to practice Guided Imagery at least once a week. The PACE group received one reminder to watch the module at least once during the six weeks after discharge. During the first review visit, a week after discharge, participants completed HADS. During the second review, six weeks after discharge, HADS and BIPROSCAB were administered. Patients were debriefed. The Control group participants were each given a DVD of the pre-discharge PACE module to honour ethical obligation.

\section{Statistical analyses}

Prognosis and psychological distress were compared across the three groups using Analyses Of Variance (ANOVA). Data obtained in the study (based on non-randomised sampling) did not fully sat- isfy the assumptions of Fisher's ANOVA. Welch ANOVA has been recommended as a robust alternative to the traditional $F$-test for samples demonstrating non-normal distribution and heterogeneity of variance (Moder, 2010; Tomarken \& Serlin, 1986). Another approach found to adjust for non-normal data is to transform the scores, for instance, into logarithmic form (Mangiafico, 2016). To analyse group differences in psychological distress across timepoints, psychological distress scores were log transformed before computing a mixed model ANOVA. In order to further delineate the pathway of impact from psychosocial intervention to prognosis, multiple linear regression analyses were carried out. All data were analysed by means of the SPSS software package.

\section{Results}

\section{Effect of psychosocial intervention on prognosis}

To determine whether prognosis differed across the groups, one-way between-subjects Welch ANOVA was computed for overall and dimension scores. These findings are presented in Table 2. It may be reiterated that higher score indicates better prognosis. There was a significant effect of the groups on overall prognosis$F(2,184.7)=113.72, p<0.001$. The effect of the groups was also significant $(p<0.001)$ for all nine dimensions of prognosis.

Games-Howell post-hoc tests were calculated to conduct pairwise mean comparisons (Table 3). For overall prognosis, the PACE

Table 2. Descriptive statistics and one-way between subjects Welch ANOVA for prognosis (BIPROSCAB) across groups.

\begin{tabular}{|c|c|c|c|c|c|}
\hline & PACE & $\begin{array}{c}M(S D) \\
\text { Relaxation }\end{array}$ & Control & $F$ & $\omega^{2}$ \\
\hline Overall prognosis & $113.45(5.78)$ & $103.05(8.92)$ & $96.92(10.64)$ & $F(2,184.7)=113.72^{* * *}$ & 0.43 \\
\hline Post-CABG affect state & $19.87(0.49)$ & $19.27(1.61)$ & $18.44(2.46)$ & $F(2147.4)=21.54^{* * *}$ & 0.12 \\
\hline Post-CABG anxiety & $22.50(2.55)$ & $19.94(3.11)$ & $18.44(3.80)$ & $F(2,193.1)=45.07^{* * *}$ & 0.22 \\
\hline Post-CABG physical pain & $13.00(2.03)$ & $11.49(2.53)$ & $9.74(2.81)$ & $F(2,194.2)=44.86^{* * *}$ & 0.22 \\
\hline Discomfort in surgical sites & $11.06(3.52)$ & $9.01(3.89)$ & $8.88(3.33)$ & $F(2,197.2)=12.00^{* * *}$ & 0.07 \\
\hline Worry about return to normalcy & $9.60(0.88)$ & $8.61(1.79)$ & $7.98(2.27)$ & $F(2,169.3)=29.94^{* * *}$ & 0.16 \\
\hline Discomfort in the leg & $8.90(1.61)$ & $7.82(2.56)$ & $7.60(2.21)$ & $F(2,190.2)=13.64^{* * *}$ & 0.08 \\
\hline CABG bio-social by-products & $9.67(0.91)$ & $9.03(1.54)$ & $8.96(1.52)$ & $F(2,184.8)=11.43^{* * *}$ & 0.07 \\
\hline Constraints in socialising & $9.97(0.30)$ & $9.57(1.09)$ & $9.26(1.48)$ & $F(2,146.0)=16.35^{* * *}$ & 0.09 \\
\hline Infection and interference to routine life & e $8.88(1.71)$ & $8.31(2.03)$ & $7.62(1.97)$ & $F(2,196.8)=11.62^{* * *}$ & 0.07 \\
\hline
\end{tabular}

Note. ${ }^{* * *} p<0.001$

Table 3. Post-hoc analyses (Games-Howell) for prognosis (BIPROSCAB) across groups.

\begin{tabular}{lccc} 
& $\mathbf{P}-\mathbf{R}$ & $\mathbf{P}-\mathbf{C}$ & $\mathbf{R}-\mathbf{C}$ \\
Overall prognosis & $10.40^{* * *}$ & $16.53^{* * *}$ & $6.13^{* * *}$ \\
Post-CABG affect state & $0.60^{* *}$ & $1.43^{* * *}$ & $0.83^{*}$ \\
\hline Post-CABG anxiety & $2.56^{* * *}$ & $4.06^{* * *}$ & $1.50^{* *}$ \\
Post-CABG physical pain & $1.51^{* * *}$ & $3.26^{* * *}$ & $1.75^{* * *}$ \\
\hline Discomfort in surgical sites & $2.05^{* * *}$ & $2.18^{* * *}$ & 0.13 \\
Worry about return to normalcy & $0.99^{* * *}$ & $1.62^{* * *}$ & 0.63 \\
\hline Discomfort in the leg & $1.08^{* *}$ & $1.30^{* * *}$ & 0.22 \\
CABG bio-social by-products & $0.64^{* *}$ & $0.71^{* * *}$ & 0.07 \\
\hline Constraints in socialising & $0.40^{* *}$ & $0.71^{* * *}$ & 0.31 \\
Infection and interference to routine life & 0.57 & $1.26^{* * *}$ & $0.69^{*}$ \\
\hline
\end{tabular}

Note. $\mathrm{P}=\mathrm{PACE}, \mathrm{R}=$ Relaxation, $\mathrm{C}=$ Control. ${ }^{*} p<0.05,{ }^{* *} p<0.01,{ }^{* * *} p<0.001$ 
group had the significantly highest mean, followed by the Relaxation group $(p<0.001)$ and the Control group $(p<0.001)$. In the dimension of 'post-CABG affect state', the PACE group had a significantly higher mean than the Relaxation group $(p<0.01)$, and the Control group $(p<0.001)$. The Relaxation group too had a significantly higher mean than the Control group $(p<0.05)$. With regard to the 'post-CABG anxiety' dimension, the mean of the PACE group was significantly higher than those of the Relaxation and Control groups $(p<0.001)$. The mean of the Relaxation group was also significantly higher than that of the Control group $(p<0.01)$. For the 'post-CABG physical pain' dimension, the PACE group had the significantly highest mean, followed by the Relaxation group $(p<0.001)$ and the Control group $(p<0.001)$. In the dimension of 'discomfort in surgical sites', the mean of the PACE group was significantly higher than those of the Relaxation and Control groups $(p<0.001)$. With regard to the dimension of 'worry about return to normalcy', the PACE group had a significantly higher mean than the Relaxation and Control groups $(p<0.001)$. For the dimension of 'discomfort in the leg', the mean of the PACE group was significantly higher than that of the Relaxation group $(p<0.01)$, and the Control group $(p<0.001)$. In the 'CABG bio-social by-products' dimension, the PACE group had a significantly higher mean than that of the Relaxation group $(p<0.01)$ and the Control group $(p<0.001)$. With regard to the dimension of 'constraints in socialising', the mean of the PACE group was significantly higher than that of the Relaxation group $(p<0.01)$, and the Control group $(p<0.001)$. In the dimension of 'infection and interference to routine life', the Control group had a significantly lower mean than the PACE group $(p<0.001)$, and the Relaxation group $(p<0.05)$.

It is evident that the PACE group showed significantly higher prognosis overall as well as in the dimensions when compared with the Relaxation and Control groups.

\section{Effect of psychosocial intervention on psychological dis- tress across time}

A 3 (groups) $\times 3$ (time-points) mixed-design ANOVA was computed using log transformed psychological distress scores to examine the effect of psychosocial intervention on psychological distress across time. The means are given in Table 4, and the trends are plotted in Figure 1. There was a significant main effect of time, $F(2,594)=299.12, p<0.001$, and group, $F(2,297)=113.46, p<0.001$. The time $\times$ group interaction effect was also significant, $F(4$, 594) $=55.47, p<0.001$.

Analyses of the simple effects of time, group-wise, revealed that in the PACE group, mean psychological distress at the presurgery point was significantly higher than at each of the two reviews $(p<0.001)$. For the Relaxation group, mean psychological distress at the pre-surgery point was significantly higher than at the two reviews $(p<0.001)$. Mean psychological distress at the first review was also significantly higher than at the second review in the Relaxation group $(p<0.001)$. Within the Control group, mean psychological distress at the pre-surgery point was significantly higher than at the first review $(p<0.01)$ and the second review $(p<0.001)$.

Considering the simple effects of group at each time-point, it was found that the three groups did not significantly differ from each other in mean psychological distress before surgery. At the first review, mean psychological distress of the PACE group was significantly lower than those of the Relaxation and Control groups $(p<0.001)$. The Relaxation group too had significantly lower mean psychological distress than the Control group $(p<0.001)$. At the second review, mean psychological distress of the PACE group was significantly lower than those of the Relaxation and Control groups $(p<0.001)$. Further, the Relaxation group had significantly lower mean psychological distress than the Control group $(p<0.001)$.

\section{Pathway of impact between psychosocial interventions and prognosis}

Multiple linear regression analyses were undertaken in two stages to identify the significant predictors of psychological distress (at different time-points) and prognosis.

\section{Stage 1}

Psychological distress-1 (measured before surgery) was regressed on age, gender, and education. The resulting model, inclusive of the three predictors, was found to be significant and explained $4 \%$ of variance in psychological distress $-1, R^{2}=0.04$, $F(3,296)=3.69, p<0.05$. Gender $(p<0.05)$ and age $(p<0.05)$ were the significant independent predictors.

Psychological distress-2 (measured at the first review) was regressed on age, gender, education, PACE, Relaxation, Control, and psychological distress-1. A significant model, consisting of

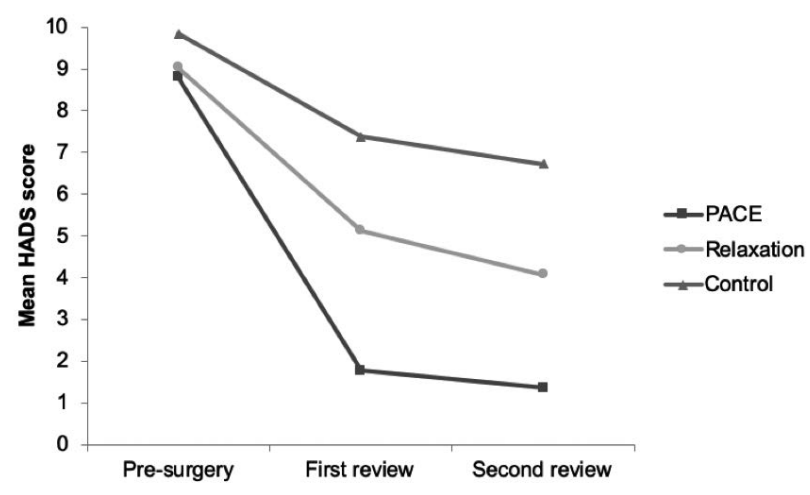

Figure 1. Mean psychological distress in the groups across time.

Table 4. Descriptive statistics for psychological distress (HADS) across groups.

\begin{tabular}{llcc} 
& & M (SD) & Control \\
Pre-surgery & PACE & Relaxation & $9.84(5.39)$ \\
First review & $8.80(4.62)$ & $9.03(4.73)$ & $7.38(3.77)$ \\
\hline Second review & $1.77(1.72)$ & $5.13(2.83)$ & $6.72(3.60)$ \\
\hline
\end{tabular}


age, gender, education, PACE, Relaxation and psychological distress-1, emerged. It explained $45 \%$ of variance in psychological distress $-2, R^{2}=0.45, F(6,293)=39.51, p<0.001$. The significant independent predictors were PACE $(p<0.001)$, Relaxation $(p<0.001)$, and psychological distress $-1(p<0.001)$.

Psychological distress-3 (measured at the second review) was regressed on age, gender, education, PACE, Relaxation, Control, psychological distress -1 , and psychological distress -2 . The resulting model, which comprised age, gender, education, PACE, Relaxation, psychological distress -1 and psychological distress -2 , was noted to be significant. It explained $55 \%$ of variance in psychological distress $-3, R^{2}=0.55, F(7,292)=51.45, p<0.001$. PACE $(p<0.001)$, Relaxation $(p<0.001)$, psychological distress -2 $(p<0.001)$, and psychological distress $-1 \quad(p<0.05)$ emerged as significant independent predictors.

Overall prognosis was regressed on age, gender, education, PACE, Relaxation, Control, psychological distress-1, psychological distress-2, and psychological distress-3. A significant model, inclusive of age, gender, education, PACE, Relaxation, psychological distress-1, psychological distress-2 and psychological distress -3 , was observed to be significant. It explained $61 \%$ of variance in overall prognosis, $R^{2}=0.61, F(8,291)=56.30, p<0.001$. Psychological distress $-3(p<0.001)$, PACE $(p<0.001)$, psychological distress $-1(p<0.01)$, and education $(p<0.05)$ were the significant independent predictors.

\section{Stage 2}

Multiple linear regression analyses were repeated using only the respective significant independent predictors of psychological distress and overall prognosis. This was meant to identify the strength of the contribution of the independent predictors to the criterion variables, and to subsequently trace the pathway of impact.

The reduced model for psychological distress -1 significantly explained $4 \%$ of variance in psychological distress $-1, R^{2}=0.04$, $F(2,297)=5.55, p<0.01$. It consisted of gender $(\beta=0.15, p<0.01)$ and age $(\beta=-0.12, p<0.05)$. This implied that being a woman or being young independently predicted high psychological distress before CABG.

The reduced model for psychological distress -2 significantly explained $44 \%$ of variance in psychological distress $-2, R^{2}=0.44$, $F(3,296)=77.11, p<0.001$. It was constituted by PACE $(\beta=-0.69$, $p<0.001)$, Relaxation $(\beta=-0.27, p<0.01)$, and psychological distress $-1 \quad(\beta=0.22, p<0.001)$. This indicated that receiving either intervention, or having low psychological distress before surgery independently predicted low psychological distress at the first review.

The reduced model for psychological distress -3 significantly explained $55 \%$ of variance in psychological distress $-3, R^{2}=0.55$, $F(4,295)=89.41, p<0.001$. It comprised psychological distress -2 $(\beta=0.48, p<0.001)$, PACE $(\beta=-0.35, p<0.001)$, Relaxation $(\beta=-$ $0.20, p<0.001)$, and psychological distress $-1 \quad(\beta=0.11, p<0.05)$. The finding suggested that having low psychological distress before surgery or at the first review, or receiving either intervention independently predicted low psychological distress at the second review.

The reduced model for overall prognosis significantly explained $60 \%$ of variance in overall prognosis, $R^{2}=0.60$, $F(4,295)=110.12, p<0.001$. It included psychological distress -3 $(\beta=-0.52, p<0.001)$, PACE $(\beta=0.30, p<0.001)$, psychological distress $-1 \quad(\beta=-0.13, p<0.01)$, and education $(\beta=0.11, p<0.01)$. The finding revealed that having low psychological distress before surgery or at the second review, receiving the PACE intervention, or having more years of education independently predicted high overall prognosis.

It is apparent that psychological distress-3 was the largest independent predictor of overall prognosis. Psychological distress -3 itself was independently predicted by the PACE and Relaxation interventions. Figures 2 and 3 illustrate the respective pathways of impact for the PACE and Relaxation interventions. Although PACE was a significant independent predictor of overall prognosis (suggesting its direct positive impact on prognosis), the greater independent contribution to overall prognosis was from psychological distress-3. Yet, PACE also significantly predicted low psychological distress-3. Thus, PACE was able to enhance prognosis by reducing overall distress at the second review, in addition to bearing a direct impact on prognosis.

The Relaxation intervention was not a significant independent predictor of overall prognosis. In the absence of a direct impact, the intervention was able to enhance prognosis only by reducing psychological distress at the second review. No pathway was identified for the Control condition, as it was statistically dropped from all multiple regression models of prediction.

\section{PACE}
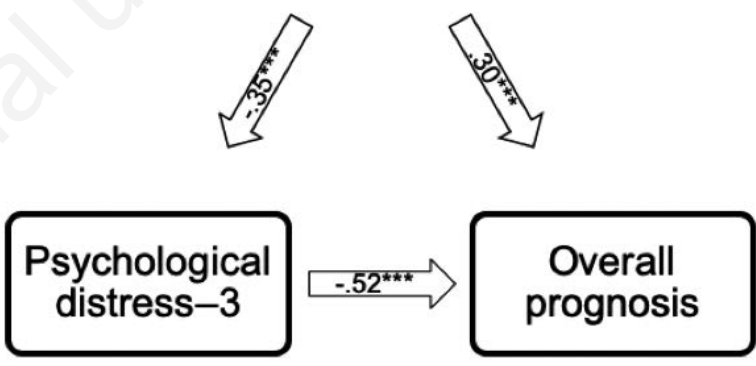

Figure 2. Pathway between the PACE intervention and prognosis of patients subjected to CABG. Arrow: Significant independent path with $\beta$ coefficient $\left({ }^{* * *} p<0.001\right)$.
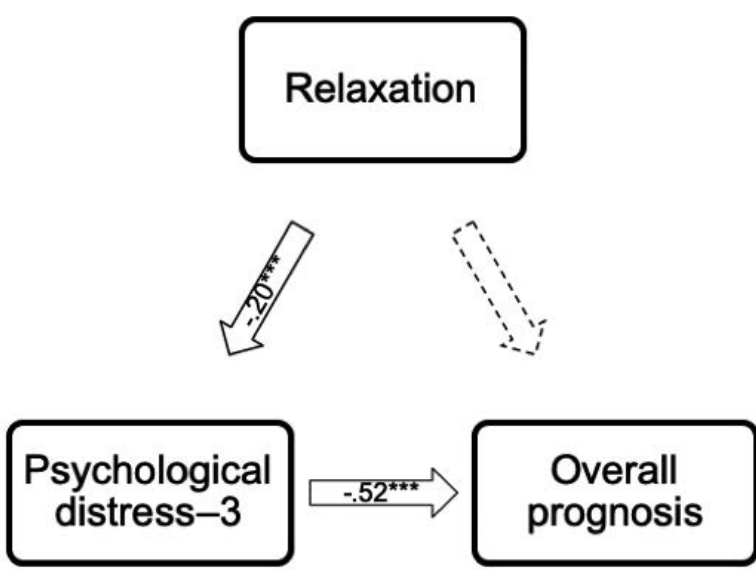

Figure 3. Pathway between the Relaxation intervention and prognosis of patients subjected to CABG. Bold Arrow: Significant independent path with $\beta$ coefficient $\left({ }^{* * *} \mathrm{p}<0.001\right)$; Dotted Arrow: Non-significant path. 


\section{Discussion}

The study sought to test two psychosocial interventions for patients undergoing CABG. We conjectured that the interventions would enhance prognosis, exhibit differential impact on psychological distress across time, and that the pathway of impact from psychosocial intervention to prognosis would be traced through psychological distress. The results partially supported the first and third hypotheses as only the PACE group consistently showed higher prognosis than the Control group, and further the PACE intervention had a strong indirect impact (through psychological distress) as well as a direct (albeit weaker) impact on prognosis. The finding of a significant interaction between group and time for psychological distress led to the acceptance of the second hypothesis. Overall, the suitability and efficacy of the unique psycho-educational intervention (PACE) was confirmed.

The dynamism of psychological distress was apparent as it varied under the joint influence of time and group. As anticipated, psychological distress declined within each group from the presurgery point to the second post-surgery review. However, psychological distress at the second review was the largest independent predictor of prognosis, confirming claims in literature that psychological distress is consequential for life after surgery (Douki et al., 2011; Thomas et al., 2016). The simple effects of group additionally showed that although psychological distress had not significantly differed across groups before surgery, significant group differences were evident at the two reviews. Pertinently, the PACE group had the lowest level of psychological distress, followed by the Relaxation group and lastly the Control group. The current psychosocial interventions thus met the fundamental goal of lowering psychological distress in patients undergoing CABG.

Prognosis in the present sample was an index of bio-physiological symptoms, psychological concerns, and social impediments after hospital discharge. These experiences characterise the periods of convalescence and return to normal activities (Daniels \& Nicoll, 2012). Since overall prognosis was noted to significantly differ across groups, the quality and speed of recovery is inferred to have substantially varied for the three patient groups despite them sharing homogenous characteristics at baseline. In terms of overall and dimension-wise prognosis, the PACE group - when compared with the Control group - occupied a more favourable position. The Relaxation group showed better prognosis than the Control group, overall and in four dimensions ('post-CABG affect state', 'postCABG anxiety', 'post-CABG physical pain', and 'infection and interference to routine life').

The two observations primarily imply that receiving psychosocial intervention enhances recovery and well-being after CABG. However, the gains of the Relaxation group in relation to the Control group were largely confined to emotional dimensions (e.g., affect, anxiety). This echoes earlier studies wherein relaxation therapy was observed to decrease distress (e.g., Firoozabadi \& Ebadi, 2014). The PACE group, conversely, showed significant improvements even in physiological domains (e.g., surgical site and leg discomforts) and interpersonal aspects (e.g., socialising) as against the Control group. In line with previous reports (e.g., Cebeci \& Çelik, 2008), the psycho-educational PACE led to a gamut of benefits across domains of recovery and well-being. Moreover, prognosis in the PACE group was significantly higher than in the Relaxation group, overall and in the dimensions except 'infection and interference to routine life'. The findings enrich literature by highlighting the differential impact of PACE and Relaxation.
The 2-part, 40-minute, audio-visual intervention of PACE brought together technical and subjective perspectives, providing virtual peer support alongside objective expert information. Theoretically thus, PACE was an adaptation of the InformationMotivation-Behavioural Skills theory (Fisher, Fisher, \& Harman, 2003). The intervention was invested on the teachable moment potential of the CABG situation. Information on capitalising social support, managing expected post-surgical problems, and resuming normal routine may have effected an attitudinal transformation that was antagonistic to the sick-role mindset and behaviour. Waight, Strodl, Sheridan, and Tesar, (2015) argued that the patients' potential for post-traumatic growth after CABG is influenced by their interpretations of change as well as their receipt of support from an acquaintance or a relative with experience of CABG. The knowledge and peer support delivered through PACE may have prompted post-traumatic growth that added up to favourable prognosis. In fact, single exposure to the modules before and after surgery were adequate to directly impact prognosis. Thus, besides its potential for superior patient outcomes, PACE was compact, accessible and aptly timed.

The Relaxation intervention, on the other hand, was capable of assuaging apprehensions through repeated exposure albeit without directly improving prognosis. The trajectory may be elucidated using the Broaden-and-Build theory (Fredrickson, 2001). The pleasant visualisation in Guided Imagery evokes positive emotions and suppresses negative affect. Periodic practice over the 6-week study time-frame may have instilled a steadily positive affect state which is ideal for broadening cognition and behaviours to promote health and well-being in the long run (Fredrickson, 2000). Relaxation may be useful in emergency surgery to relieve distress, and in contexts where patients are adequately informed about CAD and $\mathrm{CABG}$ yet require therapeutic support to overcome the distress of surgery.

The significantly highest psychological distress and lowest prognosis in the Control group critically point out the underwhelming effect of standard hospital treatment during $\mathrm{CABG}$, resulting in mediocre outcomes. Hospitals in the study did indeed engage educational strategies such as broadcasting informational videos and placing informational hand-outs in waiting rooms. Yet, since the Control group consistently demonstrated lower prognosis than the PACE group and had not significantly contributed to distress reduction or prognosis improvement, it may be inferred that the PACE intervention was more effective than existing practices of patient education and counselling.

Certain study limitations must be acknowledged to aid further research and practice. The findings are confined to a 6-week postdischarge period. It cannot be ascertained whether the gains may be generalised to when patients return to a fully functional and working life. Secondly, post-discharge psychosocial intervention was aided through CD/DVD, which may not have matched up to direct professional intervention. Nonetheless, as Correa-Rodríguez et al. (2020) emphasised the need for periodic psychosocial evaluation, the present study provided continuous data (three timepoints) across the pre- and post-operative contexts while also comparing three intervention conditions.

Psychological assessment and intervention thus merit integration into routine surgical care. The peer-based educational approach is an optimal resource for patient communication in large healthcare systems that are limited by health manpower (Aswathy, Unnikrishnan, Kalra, \& Leelamoni, 2013). In future, provision of continuous monitored intervention and repeated measurement to observe the impact after six weeks may be undertaken during outpatient reviews that generally occur at 6-month intervals. 


\section{References}

Aswathy, S., Unnikrishnan, A. G., Kalra, S., \& Leelamoni, K. (2013). Peer support as a strategy for effective management of diabetes in India. Indian Journal of Endocrinology and Metabolism, 17, 5-7. https://doi.org/10.4103/2230-8210. 107790

Bjelland, I., Dahl, A. A., Haug, T. T., \& Neckelmann, D. (2002). The validity of the Hospital Anxiety and Depression Scale: An updated literature review. Journal of Psychosomatic Research, 52, 69-77. https://doi.org/10.1016/S0022-3999(01)00296-3

Broadbent, E., Petrie, K. J., Ellis, C. J., Ying, J., \& Gamble, G. (2004). A picture of health-myocardial infarction patients' drawings of their hearts and subsequent disability: A longitudinal study. Journal of Psychosomatic Research, 57, 583-587. https://doi.org/10.1016/j.jpsychores.2004.03.014

Cebeci, F., \& Çelik, S. Ş. (2008). Discharge training and counselling increase self-care ability and reduce postdischarge problems in CABG patients. Journal of Clinical Nursing, 17, 412-420. https://doi.org/10.1111/j.1365-2702.2007.01952.x

Chaudhury, S., Saini, R., Bakhla, A. K., \& Singh, J. (2016). Depression and anxiety following Coronary Artery Bypass Graft: Current Indian scenario. Cardiology Research and Practice, 2016, 2345184. https://doi.org/10.1155/2016/2345184

Colom, F. (2011). Keeping therapies simple: Psychoeducation in the prevention of relapse in affective disorders. British Journal of Psychiatry, 198, 338-340. https://doi.org/10.1192/bjp.bp.110.090209

Correa-Rodríguez, M., Ejheisheh, M. A., Suleiman-Martos, N., Membrive-Jiménez, M. J., Velando-Soriano, A., SchmidtRioValle, J., \& Gómez-Urquiza, J. L. (2020). Prevalence of depression in Coronary Artery Bypass surgery: A systematic review and meta-analysis. Journal of Clinical Medicine, 9, 909. https://doi.org/10.3390/jcm9040909

Daniels, R., \& Nicoll, L. H. (2012). Contemporary MedicalSurgical Nursing (2 $2^{\text {nd }}$ ed.). Delmar Cengage Learning.

Douki, Z. E., Vaezzadeh, N., Shahmohammadi, S., Shahhosseini, Z., Tabary, S. Z., Mohammadpour, R. A., \& Esmaeeli, M. (2011). Anxiety before and after Coronary Artery Bypass Grafting surgery: Relationship to QOL. Middle-East Journal of Scientific Research, 7, 103-108.

Drapeau, A., Marchand, A., \& Beaulieu-Prévost, D. (2012). Epidemiology of psychological distress. In L. L'Abate (Ed.), Mental Illnesses: Understanding, Prediction and Control (pp. 105-134). https://doi.org/10.5772/30872.

Fathi, M., Alavi, S. M., Joudi, M., Joudi, M., Mahdikhani, H., Ferasatkish, R., Bakhshandeh, H., \& Nooghabi, M. J. (2014). Preoperative anxiety in candidates for heart surgery. Iranian Journal of Psychiatry and Behavioral Sciences, 8, 90-96.

Firoozabadi, M. D., \& Ebadi, A. (2014). Effect of relaxation on postoperative pain in patients after Coronary Artery Bypass Grafting (CABG) surgery. NationalPark-Forschung in Der Schweiz (Switzerland Research Park Journal), 103, 185-191.

Fisher, W. A., Fisher, J. D., \& Harman, J. (2003). The InformationMotivation-Behavioral skills model: A general Social Psychological approach to understanding and promoting health behavior. In J. Suls, \& K. A. Wallston (Eds.), Social Psychological Foundations of Health and Illness (pp. 82-106). Blackwell Publishing.

Fredrickson, B. L. (2000). Cultivating positive emotions to optimize health and well-being. Prevention \& Treatment, 3. http://tinyurl.com/o28me3w.

Fredrickson, B. L. (2001). The role of positive emotions in Positive Psychology: The Broaden-and-Build theory of positive emotions. American Psychologist, 56, 218-226. https://doi.org/10.1037/0003-066X.56.3.218

Gaziano, T. A., Bitton, A., Anand, S., Abrahams-Gessel, S., \& Murphy, A. (2010). Growing epidemic of Coronary Heart Disease in low- and middle-income countries. Current Problems in Cardiology, 35, 72-115. https://doi.org/10.1016/j.cpcardiol.2009.10.002

Hariharan, M., Thomas, M., \& Rana, S. (2017). Development and preliminary testing of the Biopsychosocial Prognosis Scale for CABG. Indian Journal of Psychological Medicine, 39, 619626.

Hilden, J., \& Habbema, J. D. F. (1987). Prognosis in medicine: An analysis of its meaning and rôles. Theoretical Medicine, 8 , 349-365. https://doi.org/10.1007/BF00489469

Mallik, S., Krumholz, H. M., Lin, Z. Q., Kasl, S. V., Mattera, J. A., Roumains, S. A., \& Vaccarino, V. (2005). Patients with depressive symptoms have lower health status benefits after Coronary Artery Bypass surgery. Circulation, 111, 271-277. https://doi.org/10.1161/01.CIR.0000152102.29293.D7

Mangiafico, S. S. (2016). Summary and Analysis of Extension Program Evaluation in $R$ (version1.18.1). Retrieved from https://rcompanion.org/handbook/

Martinsen, R., \& Moen, A. (2010). Unmet information needs and communication needs in the intermediate recovery from Coronary Artery Bypass surgery. Nordic Journal of Nursing Research, 30, 15-19.

Miller, D. W. Jr. (1977). Benefits of Coronary Artery Bypass surgery. Archives of Internal Medicine, 137, 1439-1446. https://doi.org/10.1001/archinte.1977.03630220073017

Moder, K. (2010). Alternatives to $F$-test in one way ANOVA in case of heterogeneity of variance (a simulation study). Psychological Test and Assessment Modeling, 52, 343-353.

Parker, R. D., \& Adams, J. (2008). Activity restrictions and recovery after open chest surgery: Understanding the patient's perspective. Baylor University Medical Center Proceedings, 21, 421-425. https://doi.org/10.1080/08998280.2008.11928442

Pedersen, S. S., Versteeg, H., Denollet, J., Cheng, J. M., Serruys, P. W., \& van Domburg, R. T. (2011). Patient-rated health status predicts prognosis following Percutaneous Coronary Intervention with drug-eluting stenting. Quality of Life Research, 20, 559-567. https://doi.org/10.1007/s11136-0109775-5

Protogerou, C., Fleeman, N., Dwan, K., Richardson, M., Dundar, Y., \& Hagger, M. S. (2015). Moderators of the effect of psychological interventions on depression and anxiety in cardiac surgery patients: A systematic review and meta-analysis. Behaviour Research and Therapy, 73, 151-164. https://doi.org/10.1016/j.brat.2015.08.004

Redelmeier, D. A., Rozin, P., \& Kahneman, D. (1993). Understanding patients' decisions: Cognitive and emotional perspectives. Journal of the American Medical Association, 270, 72-76. https://doi.org/10.1001/jama.1993.035100 10078034

Sipahi, I., Akay, M. H., Dagdelen, S., Blitz, A., \& Alhan, C. (2014). Coronary Artery Bypass Grafting vs Percutaneous Coronary Intervention and long-term mortality and morbidity in multivessel disease: Meta-analysis of randomized clinical trials of the arterial grafting and stenting era. JAMA Internal Medicine, 174, 223-230. https://doi.org/10.1001/jamainternmed.2013. 12844 
Smith, G. (2012). An introduction to psychological interventions. In G. Smith (Ed.), Psychological Interventions in Mental Health Nursing (pp. 1-23). Open University Press.

Thomas, M., Hariharan, M., \& Rana, S. (2016). Psychological distress as predictor of adherence and prognosis among patients undergoing Coronary Artery Bypass Grafting. Journal of the Indian Academy of Applied Psychology, 42, 161-171.

Tomarken, A. J., \& Serlin, R. C. (1986). Comparison of ANOVA alternatives under variance heterogeneity and specific noncentrality structures. Psychological Bulletin, 99, 90-99.

Upton, D., \& Solowiej, K. (2010). Pain and stress as contributors to delayed wound healing. Wound Practice and Research, 18, 114-122.

van Dixhoorn, J., \& White, A. (2005). Relaxation therapy for rehabilitation and prevention in Ischemic Heart Disease: A systemic review and meta-analysis. European Journal of
Preventive Cardiology, 12, 193-202.

Waight, C. A., Strodl, E., Sheridan, J., \& Tesar, P. (2015). Posttraumatic growth in post-surgical Coronary Artery Bypass Graft patients. Health Psychology Open, 2, 1-9. https://doi.org/10.1177/2055102915571370

World Health Organization. (2015). Noncommunicable diseases Fact sheet. http://tinyurl.com/pdb8w2t. Accessed 20 April 2016.

Wulsin, L. (2012). Psychological challenges of coping with Coronary Artery Disease. In E. A. Dornelas (Ed.), Stress Proof the Heart: Behavioral Interventions for Cardiac Patients (pp. 9-24). Springer.

Zigmond, A. S., \& Snaith, R. P. (1983). The Hospital Anxiety and Depression Scale. Acta Psychiatrica Scandinavica, 67, 361370. https://doi.org/10.1111/j.1600-0447.1983.tb09716.x 